\title{
Improved Medium for Selecting Nitrate-Nonutilizing (nit) Mutants of Verticillium dahliae
}

\author{
Nadia Korolev and Talma Katan
}

Department of Plant Pathology, Agricultural Research Organization, The Volcani Center, P.O. Box 6, Bet Dagan 50250, Israel. Accepted for publication 17 June 1997.

\begin{abstract}
Korolev, N., and Katan, T. 1997. Improved medium for selecting nitratenonutilizing (nit) mutants of Verticillium dahliae. Phytopathology 87: 1067-1070.

Nitrate-nonutilizing (nit) mutants are commonly used to determine vegetative compatibility between isolates of Verticillium dahliae by complementation (heterokaryon) testing. These mutants emerge spontaneously as chlorate-resistant sectors growing out of partially restricted, wild-type colonies on chlorate-amended media. The commonly used chlorate media

ed with potassium chlorate. nit mutants recovered on these media constituted 10 to $36 \%$ (on MMC) and 25 to $45 \%$ (on CMC) of the apparently resistant sectors. An improved water agar chlorate medium (WAC) is described that is more effective for selecting chlorate-resistant nit mutants. WAC medium consists of agar (2\%), glucose $(0.02 \%)$, and potassium chlorate (2 to 5\%). On WAC, growth of most $V$. dahliae isolates was strongly inhibited, and 66 to $100 \%$ (average $>80 \%$ ) of the chlorate-resistant sectors formed were nit mutants. Most mutants were characterized as nitl, and about $6 \%$ as NitM.
\end{abstract} are based on minimal medium (MMC) or cornmeal agar (CMC), amend-
Identification of vegetative compatibility groups has proven to be an effective approach for studying genetic relationships in anamorphic populations of several fungi, including the important and widespread plant pathogen Verticillium dahliae $(17,18,22,23)$. Nitrate-nonutilizing (nit) mutants are the most commonly used in complementation (heterokaryon) tests, because they arise spontaneously at a high frequency and can be easily recovered as chlorate-resistant sectors or clones (7). Usually, minimal medium amended with potassium chlorate (MMC), as described for Fusarium oxysporum $(5,21)$, is suitable for other species and genera as well $(10,13,14,20)$. This medium, along with chlorate cornmeal agar (CMC), has been commonly used for generating nit mutants from $V$. dahliae and $V$. albo-atrum $(1,3,4,9,11,12,24-26)$. However, difficulties in isolating mutants from some strains $(3,9,11,12,25,26)$ and the need to purify nit mutants from apparently chlorate-resistant sectors by isolating single germinating conidia or hyphal tips $(4,9)$ have been reported. In an ongoing study of genetic diversity in populations of $V$. dahliae in Israel (16), we have encountered similar difficulties; the proportion of nit mutants recovered from resistant sectors developing on the common chlorate media is relatively small. Consequently, repeated inoculation on chlorate media and further purification steps have been necessary to obtain satisfactory numbers of nit mutants. The relatively low recovery of nit mutants among the sectors tested, possibly resulting from the difficulties involved in visually distinguishing resistant sectors from the wild-type parental colony on MMC and CMC, led us to look for a more effective chlorate medium. We hypothesized that a medium with a reduced nutrient level would be more restrictive to the radial growth of wildtype colonies, thereby allowing for the more conspicuous appearance of mutant sectors. The objective of this work was to formulate a chlorate medium that would improve the recovery of nit mutants from V. dahliae.

Corresponding author: T. Katan; E-mail address: vpptlg@ volcani.agri.gov.il

Publication no. P-1997-0728-01R

(C) 1997 The American Phytopathological Society

\section{MATERIALS AND METHODS}

Isolation of Verticillium from plants and soil. $V$. dahliae Kleb. was isolated from cotton (Gossypium hirsutum L.), potato (Solanum tuberosum L.), eggplant (Solanum melongena L.), Chrysanthemum spp., other host plants, and soil. Plants showing symptoms of Verticillium wilt were collected from fields and greenhouses. Pieces of vascular tissue excised from basal stem segments were surfacesterilized with $0.5 \% \mathrm{NaOCl}$ for $1 \mathrm{~min}$, rinsed in sterile distilled water, and placed on either Czapek-Dox agar (CDA; Biolife, Milan, Italy) amended with streptomycin or penicillin sulfate $(100 \mathrm{mg} /$ liter), or Verticillium-selective medium (2). Plates were incubated at $24^{\circ} \mathrm{C}$ for 5 to 7 days until colonies with verticillately branched conidiophores developed around the stem tissue. The soil dilution method was used to isolate Verticillium from naturally infested soil. Each fresh sample of soil from the field or plant rhizosphere was mixed thoroughly, and then triplicate 5-g subsamples were suspended in $250 \mathrm{ml}$ of sterile distilled water and shaken for $20 \mathrm{~min}$. Soil suspension ( $0.3 \mathrm{ml}$ per plate, 10 plates per subsample) was spread on alcohol agar (19) and incubated for 10 to 12 days at room temperature. Colonies of $V$. dahliae with black microsclerotia could be distinguished against a white background. Conidia from colonies were streaked on CDA and incubated overnight at $24^{\circ} \mathrm{C}$. Germinated conidia were individually transferred to fresh CDA and maintained at $24^{\circ} \mathrm{C}$. Out of 200 monoconidial isolates used, about $37 \%$ were obtained from cotton or cotton field soil, $47 \%$ from potato or potato field soil, and the remainder from other plant species. Subcultures of all isolates were stored on CDA at 5 to $7^{\circ} \mathrm{C}$, and some were also stored on sterilized, dried Whatman no. 54 filter paper (Whatman International, Ltd., Maidstone, England) (6).

Generation and characterization of nit mutants. Three potassium chlorate-containing media in 9-cm-diameter petri plates were used to generate nit mutants: cornmeal agar with $0.02 \%$ glucose (CMC) (4), minimal medium (MMC) $(5,21)$, and water agar ( $2 \%$ ) with $0.02 \%$ glucose (WAC). The media were amended with 20 to $50 \mathrm{~g} /$ liter of potassium chlorate. Mycelial agar plugs of $V$. dahliae isolates were removed and placed on the chlorate media, either in the center of the agar surface or at three to six separate points, and the plates were incubated at $24^{\circ} \mathrm{C}$. Chlorate-resistant 
sectors that grew out of the developing colonies, evident after 10 to 28 days, were transferred to CDA plates $(5 \mathrm{~cm}$ in diameter). $\mathrm{Sec}-$ tors that grew on CDA as expansive colonies with a thin, nonaerial mycelium were considered nit mutants. The CDA medium amended with $\mathrm{NaNO}_{2}(0.5 \mathrm{~g} /$ liter $)$ or hypoxanthine $(0.2 \mathrm{~g} /$ liter $)$ was used for partial phenotyping of the nit mutants (5). Mutants that grew sparsely on hypoxanthine were classified as NitM, whereas mutants that grew profusely on hypoxanthine were classified as nitl.

Recovery of nit mutants from CMC. To determine the effectiveness of CMC in generating nit mutants from various isolates, four monoconidial subcultures were prepared from each of 50 isolates and used as replicates in four experiments (one subculture per experiment). Mycelial plugs from the $V$. dahliae subcultures were placed in the center of CMC plates containing 2.0 or $2.5 \%$ chlorate, three to five plates per isolate, and incubated for 2 to 4 weeks at $24^{\circ} \mathrm{C}$. Radial growth of wild-type colonies was only partially retarded on this medium, although growth was appressed with little aerial mycelium and fewer microsclerotia. Chlorate-resistant sectors appeared as slightly faster-growing mycelium at the colony perimeter, or as patches of more dense, aerial growth scattered about the colony. A transfer loop was used to remove conidia from such sectors or patches and streak them on CDA. The resultant growth was often a mosaic of both wild-type and thin mycelium, requiring repeated purification steps. Only colonies that grew on CDA as expansive, thin mycelium were considered nit mutants.

Recovery of nit mutants from three chlorate media. In addition to CMC, MMC and WAC were used at two potassium chlorate concentrations (20 and $50 \mathrm{~g} /$ liter). Three $V$. dahliae isolates, one from eggplant and two from cotton, were used. Mycelial plugs of each isolate were inoculated at three points in each plate, three plates for every medium-isolate combination. Chlorate-resistant sectors or patches were subcultured on CDA after 10 to 21 days of incubation, and the proportion of nit mutants was determined 5 days later.

Recovery of nit mutants from WAC. WAC is a very simple medium consisting of agar, glucose, and potassium chlorate (20 or $50 \mathrm{~g} /$ liter). The generation of nit and NitM mutants was investigated using 200 different isolates in four experiments. Two types of colony growth and sector formation were observed on this medium, depending on the isolates tested and their chlorate sensitivity. Growth of most wild-type isolates was limited, reaching 3 to $4 \mathrm{~mm}$ around the inoculum plug after 10 to 14 days of incubation.

Complementation (heterokaryon) tests. Complementation between phenotypically distinct nit mutants was tested on CDA in 5$\mathrm{cm}$ petri plates. Three mutants were inoculated per plate, 1 to 1.5 $\mathrm{cm}$ apart in a triangular pattern, and incubated at $24^{\circ} \mathrm{C}$. Complementation was evident by the formation of a dense, wild-type mycelium where two mutants had met and formed a stable heterokaryon, usually 14 to 28 days after inoculation.

\section{RESULTS}

Recovery of nit mutants from CMC. An average of $1.6 \mathrm{sec}-$ tors per colony was obtained, and about $35 \%$ of the sectors tested

TABLE 1. Recovery of nitrate nonutilizing (nit) mutants from 50 isolates ${ }^{\mathrm{a}}$ of Verticillium dahliae on chlorate cornmeal agar (CMC)

\begin{tabular}{|c|c|c|c|c|c|}
\hline \multirow[b]{2}{*}{ Replication $^{\mathrm{a}}$} & \multirow[b]{2}{*}{$\begin{array}{l}\text { No. of } \\
\text { sectors }\end{array}$} & \multicolumn{2}{|c|}{ nit mutants } & \multicolumn{2}{|c|}{ NitM } \\
\hline & & No. & $\begin{array}{l}\% \text { total } \\
\text { sectors }\end{array}$ & No. & $\begin{array}{c}\% \text { nit } \\
\text { mutants }\end{array}$ \\
\hline 1 & 523 & 200 & 38 & 21 & 10.5 \\
\hline 2 & 578 & 168 & 29 & 14 & 8.3 \\
\hline 3 & 594 & 219 & 37 & 14 & 6.4 \\
\hline 4 & 530 & 200 & 38 & 17 & 8.5 \\
\hline Total/mean $( \pm \mathrm{SD})$ & 2,225 & 787 & $35.5 \pm 4.3$ & 66 & $8.4 \pm 1.6$ \\
\hline
\end{tabular}

a Each isolate was tested four times using a different monoconidial subculture (replication) in each of four experiments (total $=200$ subcultures). were nit mutants (Table 1). Among the nit mutants, $8.4 \%$ were classified as NitM, whereas the majority were nit1. Some nit mutants grew very slowly on CDA amended with nitrite, or developed leaky, rather than expansive, thin mycelium on this medium. Consequently, they were also regarded as nitl mutants, whereas no nit 3 mutants could be recognized. All tested nit mutants exhibited wild-type growth on potato dextrose agar (PDA).

Recovery of nit mutants from three chlorate media. Neither sectoring nor the proportion of nit mutants were consistently increased by the higher chlorate concentration (Table 2), but further purification of nit mutants from mixed sectors was easier, because growth of parental colonies was more inhibited. Although fewer sectors were induced on WAC than on CMC or MMC, the proportion of nit mutants recovered from these sectors and their actual number were significantly higher (Table 2). These results suggested that WAC should be more effective than CMC or MMC in generating nit mutants, hence, this potential was further evaluated.

Recovery of nit mutants from WAC. From the restricted colony, resistant sectors appeared as a thin, fast-growing mycelium gradually surrounding the inoculum to form an asymmetric, ovalshaped colony. When conidia from such sectors were streaked onto CDA, they almost always gave rise to typical, sparsely growing nit mutant colonies. With most isolates, nit mutants were, thus, recovered from 70 to $100 \%$ of the inoculum plugs. Some isolates that were less sensitive to the chlorate concentration used grew on WAC initially as a thin colony whose radial growth was only partially reduced. On this background, resistant sectors appeared later as dense, asymmetric mycelial patches around the inoculum. Conidial transfers from such patches gave rise to mixed wild-type and mutant growth on CDA. In about $10 \%$ of the isolates tested, chlorate-resistant nitrate-utilizing (crn) sectors appeared frequently,

TABLE 2. Recovery of nitrate nonutilizing (nit) mutants from three isolates of Verticillium dahliae on three chlorate-containing media

\begin{tabular}{|c|c|c|c|c|c|}
\hline Medium $^{\mathrm{a}}$ & $\begin{array}{c}\mathrm{KClO}_{3} \\
(\%)\end{array}$ & Isolate & $\begin{array}{c}\text { No. of } \\
\text { sectors }^{b}\end{array}$ & $\begin{array}{c}\text { nit mutants } \\
(\%)\end{array}$ & \\
\hline \multirow[t]{8}{*}{ CMC } & 2 & ep8 & 12 & 25 & \\
\hline & & $\cot 30$ & 16 & 38 & \\
\hline & & $\cot 66$ & 17 & 35 & \\
\hline & & Mean & 15.0 & 32.7 & \\
\hline & 5 & ep8 & 12 & 25 & \\
\hline & & $\cot 30$ & 11 & 36 & \\
\hline & & $\cot 66$ & 20 & 45 & \\
\hline & & Mean & 14.3 & 35.3 & \\
\hline \multirow[t]{8}{*}{ MMC } & 2 & ep8 & 8 & 25 & \\
\hline & & $\cot 30$ & 10 & 10 & \\
\hline & & $\cot 66$ & 19 & 32 & \\
\hline & & Mean & 12.3 & 22.3 & \\
\hline & 5 & ep8 & 14 & 36 & \\
\hline & & $\cot 30$ & 18 & 22 & \\
\hline & & $\cot 66$ & 11 & 36 & \\
\hline & & Mean & 14.3 & 31.3 & \\
\hline \multirow[t]{8}{*}{ WAC } & 2 & ep8 & 12 & 100 & \\
\hline & & $\cot 30$ & 10 & 100 & \\
\hline & & $\cot 66$ & 11 & 64 & \\
\hline & & Mean & 11.0 & 88.0 & \\
\hline & 5 & ep8 & 9 & 100 & \\
\hline & & $\cot 30$ & 11 & 91 & \\
\hline & & $\cot 66$ & 12 & 83 & \\
\hline & & Mean & 10.7 & 91.3 & \\
\hline \multicolumn{6}{|c|}{ Analysis of variance } \\
\hline Source & df & $F$ & $P$ & $F$ & $P$ \\
\hline Medium (M) & 2 & 1.67 & 0.228 & 50.54 & 0.0001 \\
\hline $\mathrm{KClO}_{3}(\mathrm{C})$ & 1 & 0.04 & 0.850 & 0.80 & 0.388 \\
\hline $\mathrm{M} \times \mathrm{C}$ & 2 & 0.23 & 0.795 & 0.13 & 0.879 \\
\hline
\end{tabular}


making repeated inoculations necessary to obtain nit mutants. Of the 200 isolates tested (Table 3), more than $80 \%$ of the sectors yielded nit mutants, but the average frequency of NitM mutants was lower (5.5\% versus $8.4 \%$ ) than that obtained on CMC (Table 1). Several hundred isolates were later treated on this medium, and all of them gave rise to nit mutants.

Stability of nit mutants. Most nit mutants were stable when grown on CDA. However, $12 \%$ of the NitM and $22 \%$ of the nit 1 mutants developed one or more sectors of prototrophic growth after 20 to 30 days.

Complementation tests. For all tested strains (except two selfincompatible ones), formation of stable heterokaryons and biochemical complementation were observed between mutants of different phenotypes (nitl and NitM) derived from the same parental strain. Complementation also occurred in about $80 \%$ of the pairings between different NitM mutants.

\section{DISCUSSION}

Cove (7) found that chlorate-resistant mutants of Aspergillus nidulans are usually also nit mutants, growing as a thin, expansive mycelium on nitrate minimal medium. Puhalla (21) illustrated the use of complementary nit mutants to test for heterokaryosis in studies of vegetative compatibility in $F$. oxysporum. The major advantages of using nit mutants are their ready recovery without mutagenesis from chlorate-containing media, and their ability to grow expansively (but sparsely) on unsupplemented minimal media. Chlorate-resistant mutants, unable to metabolize nitrate (and chlorate), emerge from the restricted parental wild-type colony as fastergrowing sectors.

Recovery of nit mutants from Verticillium using this method has been more difficult than from other fungal species, because chlorate does not greatly restrict the growth of many strains $(4,9,26)$ that also show considerable variation in growth and sectoring patterns when grown on the commonly used media $(4,9,11)$. Chlorate media used by various researchers have been based on minimal medium $(1,3,4,9,24-26)$, cornmeal agar $(1,3,4,11,12,26)$, PDA $(3,26)$, and supplemented minimal medium (26), with chlorate concentrations ranging from 1.5 to $3 \%$. Although growth of aerial mycelium is usually suppressed on such media, radial growth is only partially reduced, and irregular colony margins may be mixed or confused with apparently resistant sectors. Conidial or mycelial transfers from such sectors frequently give rise to wild-type or mixed growth instead of the nit phenotype, as also often occurs with transfers from aerial hyphae that grow as patches on top of the parental colony $(1,3,4,9,11)$. Additional transfers are necessary, in such cases, to purify nit mutants from other components of the mixed growth $(4,9)$. Neither sectoring nor the proportion of nit mutants were consistently increased by raising the chlorate concentration in the media from 2 to $5 \%$, although further purification of nit mutants required fewer subculturing steps.

The $200 \mathrm{~V}$. dahliae isolates included in this study varied considerably in sectoring frequency. About $10 \%$ of the isolates formed very few sectors, and repeated inoculations on chlorate media (sometimes as many as 25 to 30) were required to generate nit mutants from them. Similar difficulties have been encountered by others $(3,9,11,12,25,26)$. The proportion of nit mutants recovered from sectors formed on MMC was 10 to $36 \%$ in our study and $34 \%$ (1) and $57 \%$ (9) in other studies, whereas the corresponding values for CMC were 25 to $45 \%$ and $40 \%$ (1), in agreement with another report that recovery was slightly better on CMC than on other media (3). Overall, these proportions are low, indicating a substantial waste of effort and materials, especially when studying large populations of Verticillium.

The WAC developed during this study offers an improved, inexpensive alternative to the media commonly used to generate nit mutants from $V$. dahliae. The proportion of nit mutants recovered from sectors formed on this medium ranged from 64 to $100 \%$, averaging over $80 \%$. Thus, WAC is about twice as effective as $\mathrm{CMC}$, and the number of sectors required for recovery of a given number of nit mutants is proportionally smaller. Furthermore, since growth of most wild-type isolates is very restricted on WAC, five or six plugs can be inoculated on one $9-\mathrm{cm}$ petri plate (as compared with one plug per plate of CMC or MMC), which was sufficient for the recovery of at least one nit mutant (usually three to five) per plate after 10 to 14 days of incubation. This difference translates into substantial savings on plates, medium, and incubation space. Incubation period can also be shortened on this medium, since nit mutants were recovered from most isolates after 10 to 14 days.

nit mutants may originate with mutations at several loci involved in nitrate reduction $(8,15)$. Most nit mutants can usually be divided into three phenotypic classes (nit1, nit3, and NitM), distinguishable by their growth response with nitrate, nitrite, and hypoxanthine as the nitrogen sources (5). In our work, $V$. dahliae was routinely grown on CDA (in which the nitrogen source is nitrate), and CDA amended with nitrite or hypoxanthine was used for partial phenotyping of the nit mutants. The availability of nitrate (in addition to the amendments) does not interfere with phenotypic identification, and distinction between nitl and NitM mutants of $V$. dahliae is more reliable on this set of media than on media used for Fusarium phenotyping (5). Only two phenotypes were identified among the approximately 3,000 mutants of $V$. dahliae generated in this and subsequent studies. Most mutants were classified as nitl, while only about $7 \%$ were characterized as NitM. In comparison with the previously reported values of 28 to $54 \%$ NitM $(3,9,12,24,25)$, this proportion is small. The difference may be related to culture age and storage conditions; we have noticed that old cultures of $V$. dahliae, especially those stored on an organic medium such as PDA, often give rise to higher proportions of NitM mutants than do freshly isolated strains maintained on CDA. Here, we worked primarily with fresh isolates. No nit3 mutants were identified in this study, although this phenotype could readily be identified among nit mutants of $V$. nigrescens using the same recovery and phenotyping procedures ( $\mathrm{N}$. Korolev, unpublished data). Several researchers have reported an inability to identify nit3 among their mutants $(3,12,24,25)$, while two others $(1,9)$ reported the occurrence of nit 3 in 11.6 and $5 \%$ of the mutants recovered. The inability of many mutants to grow on the nitrite-amended media used to distinguish between nit1 and nit3 phenotypes has been suggested as an explanation for the failure to identify nit 3 mutants $(3,25)$. Similarly, some of our mutants that exhibited very slow or leaky growth on nitrite-amended CDA were classified as nitl, because they did not develop a thin, expansive mycelium on this medium.

In addition to the 200 isolates documented here, the new WAC medium has been used with about 300 additional isolates of $V$. dahliae, and nit mutants were obtained from all of them. The WAC medium has proven suitable for generating nit mutants from other Verticillium species as well (N. Korolev, unpublished data) and should be useful in applications involving vegetative compatibility determinations amongst large populations of $V$. dahliae and other species.

TABLE 3. Recovery of nitrate nonutilizing (nit) mutants from 200 isolates of Verticillium dahliae on chlorate water agar (WAC)

\begin{tabular}{|c|c|c|c|c|c|}
\hline \multirow[b]{2}{*}{ Experiment $^{\mathrm{a}}$} & \multirow[b]{2}{*}{$\begin{array}{l}\text { No. of } \\
\text { sectors }\end{array}$} & \multicolumn{2}{|c|}{ nit mutants } & \multicolumn{2}{|c|}{ NitM } \\
\hline & & No. & $\begin{array}{l}\% \text { total } \\
\text { sectors }\end{array}$ & No. & $\begin{array}{c}\% \text { nit } \\
\text { mutants }\end{array}$ \\
\hline 1 & 161 & 140 & 87 & 3 & 2.1 \\
\hline 2 & 168 & 147 & 88 & 15 & 10.2 \\
\hline 3 & 159 & 125 & 79 & 10 & 8.0 \\
\hline 4 & 115 & 109 & 95 & 2 & 1.8 \\
\hline Total/mean $( \pm \mathrm{SD})$ & 603 & 521 & $87.0 \pm 6.6$ & 30 & $5.5 \pm 4.2$ \\
\hline
\end{tabular}

${ }^{\text {a }}$ Fifty isolates per experiment. 


\section{ACKNOWLEDGMENTS}

This research was supported, in part, by the Science Program of Israel Ministry of Immigration; the research fund of the Chief Scientist of the Ministry of Agriculture, Israel; and the U.S.-Israel Binational Agricultural R\&D fund (BARD). Contribution from the ARO, no. 2052-E, 1997 series.

\section{LITERATURE CITED}

1. Akimov, G. I., and Portenko, L. G. 1996. Studying vegetative compatibility in Verticillium dahliae Kleb. with non nitrate utilizing mutants. (In Russian.) Genetika 32:211-217.

2. Ausher, R., Katan, J., and Ovadia, S. 1975. An improved selective medium for the isolation of Verticillium dahliae. Phytoparasitica 3:133-137.

3. Chen, W. 1994. Vegetative compatibility groups of Verticillium dahliae from ornamental woody plants. Phytopathology 84:214-219.

4. Correll, J. C., Gordon, T. R., and McCain, A. H. 1988. Vegetative compatibility and pathogenicity of Verticillium albo-atrum. Phytopathology 78:1017-1021.

5. Correll, J. C., Klittich, C. J. R., and Leslie, J. F. 1987. Nitrate nonutilizing mutants of Fusarium oxysporum and their use in vegetative compatibility tests. Phytopathology 77:1640-1646.

6. Correll, J. C., Puhalla, J. E., and Schneider, R. W. 1986. Identification of Fusarium oxysporum f. sp. apii on the basis of colony size, virulence, and vegetative compatibility. Phytopathology 76:396-400.

7. Cove, D. J. 1976. Chlorate toxicity in Aspergillus nidulans: The selection and characterisation of chlorate resistant mutants. Heredity 36:191-203.

8. Cove, D. J. 1979. Genetic studies of nitrate assimilation in Aspergillus nidulans. Biol. Rev. 54:291-327.

9. Daayf, F., Nicole, M., and Geiger, J. P. 1995. Differentiation of Verticillium dahliae populations on the basis of vegetative compatibility and pathogenicity on cotton. Eur. J. Plant Pathol. 101:69-79.

10. Hawthorne, B. T., and Rees-George, J. 1996. Use of nitrate non-utilizing mutants to study vegetative incompatibility in Fusarium solani (Nectria haematococca), especially members of mating populations I, V and VI. Mycol. Res. 100:1075-1081.

11. Joaquim, T. R., and Rowe, R. C. 1990. Reassessment of vegetative compatibility relationships among strains of Verticillium dahliae using nitrate-nonutilizing mutants. Phytopathology 80:1160-1166.
12. Joaquim, T. R., and Rowe, R. C. 1991. Vegetative compatibility and virulence of strains of Verticillium dahliae from soil and potato plants. Phytopathology 81:552-558.

13. Katan, T., and Shabi, E. 1996. Vegetative compatibility among isolates of Colletotrichum gloeosporioides from almond in Israel. Eur. J. Plant Pathol. 102:597-600.

14. Kedera, C. J., Leslie, J. F., and Claflin, L. E. 1994. Genetic diversity of Fusarium section Liseola (Gibberella fujikuroi) in individual maize stalks. Phytopathology 84:603-607.

15. Klittich, C. J. R., and Leslie, J. F. 1988. Nitrate reduction mutants of Fusarium moniliforme (Gibberella fujikuroi). Exp. Mycol. 12:289-294.

16. Korolev, N., Bao, J. R., Katan T., and Katan, J. 1995. Vegetative compatibility groups of Verticillium dahliae in Israel. (Abstr.) Phytoparasitica 23:257.

17. Leslie, J. F. 1993. Fungal vegetative compatibility. Annu. Rev. Phytopathol. 31:127-150.

18. Leslie, J. F. 1996. Fungal vegetative compatibility-Promises and prospects. Phytoparasitica 24:3-6.

19. Nadakavukaren, M. J., and Horner, C. E. 1959. An alcohol agar medium selective for determining Verticillium microsclerotia in soil. Phytopathology 49:527-528.

20. Newton, A. C., and Caten, C. E. 1988. Auxotrophic mutants of Septoria nodorum isolated by direct screening and by selection for resistance to chlorate. Trans. Br. Mycol. Soc. 90:199-207.

21. Puhalla, J. E. 1985. Classification of strains of Fusarium oxysporum on the basis of vegetative compatibility. Can. J. Bot. 63:179-183.

22. Puhalla, J. E., and Hummel, M. 1983. Vegetative compatibility groups within Verticillium dahliae. Phytopathology 73:1305-1308.

23. Rowe, R. C. 1995. Recent progress in understanding relationships between Verticillium species and subspecific groups. Phytoparasitica 23:31-38.

24. Strausbaugh, C. A. 1993. Assessment of vegetative compatibility and virulence of Verticillium dahliae isolates from Idaho potatoes and tester strains. Phytopathology 83:1253-1258.

25. Strausbaugh, C. A., Schroth, M. N., Weinhold, A. R., and Hancock, J. G. 1992. Assessment of vegetative compatibility of Verticillium dahliae tester strains and isolates from California potatoes. Phytopathology 82:61-68.

26. Subbarao, K. V., Chassot, A., Gordon, T. R., Hubbard, J. C., Bonello, P., Mullin, R., Okamoto, D., Davis, R. M., and Koike, S. T. 1995. Genetic relationships and cross pathogenicities of Verticillium dahliae isolates from cauliflower and other crops. Phytopathology 85:1105-1112. 